\title{
Reproduction and Development of Laboratory and Wild House Dust Mites (Acari: Pyroglyphidae) and Their Relationship to the Natural Dust Ecosystem
}

\author{
B. J. HART, ${ }^{1}$ D. CROWTHER, ${ }^{2}$ T. WILKINSON,${ }^{2,3}$ P. BIDDUlPh,${ }^{2,4}$ M. UCCI ${ }^{4}$ S. PRETLOVE, ${ }^{5}$ \\ I. RIDLEY, ${ }^{4}$ AND T. ORESZCZYN ${ }^{4}$
}

J. Med. Entomol. 44(4): 568-574 (2007)

\begin{abstract}
Life histories of "wild" house dust mites, Dermatophagoides pteronyssinus (Trouessart) (Acari: Pyroglyphidae), were compared with laboratory cultures by using a diet consisting of skin and dust or a laboratory diet consisting of dried liver and yeast. Under constant conditions of $25^{\circ} \mathrm{C}$ and $75 \%$ $\mathrm{RH}$, fecundity and rate of reproduction were higher in laboratory cultures on both diets compared with wild mites. There were also trends for a shorter prereproductive period and more rapid egg development of laboratory mites compared with wild mites. Overall, there was little effect of diet on either strain of mites at $75 \% \mathrm{RH}$. At low RH (64\%), fecundity was significantly lower (for both strains on both diets), and there were also trends for longer prereproductive period, reduced rate of reproduction, reduced adult survival, prolonged egg and juvenile development, or a combination compared with $75 \% \mathrm{RH}$. Additionally egg and juvenile mortality were significantly higher on the liver and yeast diet. Overall, the skin and dust diet favored both strains of mites at $64 \% \mathrm{RH}$. On the liver and yeast diet at $64 \% \mathrm{RH}$, wild mite adults performed significantly better than laboratory mites, and egg mortality was lower. These results suggest that laboratory mites have stronger reproduction and development than wild mites, except when under environmental stress and that diet is a significant factor, particularly in suboptimal conditions. This could have important implications for predictive models of house dust mite populations in their natural habitat. Ideally, such models should be developed using data from wild dust mite populations reared on a natural diet.
\end{abstract}

KEY WORDS Dermatophagoides pteronyssinus, wild populations, life history

Population models to predict house dust mite, Dermatophagoides pteronyssinus (Trouessart) (Acari: Pyroglyphidae), populations in the home are currently under development (Pretlove et al. 2001, 2005; Crowther et al. 2006, Biddulph et al. 2007). Their aim is to assist in the effective control of mites by manipulating the temperature and relative humidity in their habitats, psychrometric conditions known to play a crucial role in their survival (Cunningham 1999, Pretlove et al. 2002). The models set out to simulate, first, psychrometric conditions in mite habitats (given climate and building characteristics), and, second, the effect of these conditions on house dust mite populations. In this way the most successful and feasible strategies for achieving psychrometric control can be

\footnotetext{
${ }^{1}$ Corresponding author: Royal Agricultural College, Stroud Road, Cirencester, Gloucestershire GL7 6JS, United Kingdom (e-mail: drbarbarahart@googlemail.com).

${ }^{2}$ Martin Centre, Cambridge University, 1 Scroope Terrace, Cambridge CB2 1PX, United Kingdom.

${ }^{3}$ Medical Entomology Centre, Barrington Rd., Shepreth, Royston SG8 6QZ, United Kingdom.

${ }^{4}$ Bartlett School of Graduate Studies, University College London WC1E 6BT, United Kingdom.

${ }^{5}$ School of Architecture \& Landscape, Kingston University, London KT1 2QJ, United Kingdom.
}

determined, whether by improving ventilation or by a combination of modifications to building design, building operation, and occupant behavior (e.g., with respect to moisture production, window opening habits). However, the population models upon which these simulations depend require mite physiology data inputs that relate to the house dust ecosystem.

Data on house dust mite reproduction and development have, until now, predominantly been obtained from mite cultures that have been reared for many years under laboratory conditions (Spieksma 1967; Blythe 1976; Dobson 1979; Gamal-Eddin et al. 1983a, 1983b, 1983c; Ho and Nadchatram 1984; Andersen 1988; Hart and Fain 1988; Arlian et al. 1990). However, in 1987, Colloff (1987a, 1987b) studied eggs from wild populations of house dust mites and suggested that they differed from eggs from laboratory populations with respect to their developmental time, mortality, and water loss. There have been no subsequent studies on wild populations of house dust mites, and no data are available on juvenile or adult physiology from wild cultures.

The principal aims of this study were therefore to obtain more detailed information on the physiology of wild house dust mite populations compared with lab- 
oratory populations and to determine the importance of wild mite data for predictive mite population models compared with existing data from long-term laboratory populations.

\section{Materials and Methods}

Mite Cultures. The laboratory strain of D. pteronyssinus had been reared for at least $10 \mathrm{yr}$ under constant laboratory conditions of $25^{\circ} \mathrm{C}$ temperature and $75 \% \mathrm{RH}$. Before experiments, they were reared under these constant hygrothermal conditions on a typical optimized liver and yeast diet of ground dried porcine liver (Oxoid, Basingstoke, UK) and brewers yeast (Holland and Barrett, Nuneaton, UK) at a 1:1 (wt:wt) ratio.

A "wild" strain of D. pteronyssinus was collected from carpet dust from a UK home in September 2004. From the time of collection, this culture was reared under fluctuating hygrothermal conditions, that is, fluctuating room temperatures and a diurnal relative humidity fluctuation of $8 \mathrm{~h}$ at $64 \% \mathrm{RH}$ and $16 \mathrm{~h}$ at $75 \%$ RH. Wild cultures were reared on a mixture of 1:0.1 (wt:wt) house dust and nondegreased (fresh) skin scales, with no addition of yeast. Experiments using these wild cultures were started in July 2005.

Mite Physiology Studies. Glass microculture vials 12 $\mathrm{mm}$ in diameter by $10 \mathrm{~mm}$ in depth were used to hold individual couples (males attached to tritonymphs) isolated from the laboratory or wild cultures for determination of adult survival and reproduction. Glue was applied around the rim of the vials to prevent escape of the mites and an equal quantity of food was added to each vial. Ten couples were used for each assay, and initially observations were made daily to determine prereproductive period and then two to three times weekly for further egg production and adult survival.

The liver and yeast diet (described above) and a skin and dust diet were used in separate experiments to determine the influence of diet on mite performance. To standardize the skin and dust diet, a stock of mattress dust was collected from the beds of a total of 20 nonsmokers, and the dust was pooled. It was then frozen at $-20^{\circ} \mathrm{C}$ for a minimum of 1 wk to kill any mites, sieved through a $500-\mu \mathrm{m}$ mesh, and then kept at room temperature for at least 1 mo before use in experiments. The aim of the latter step was to enable recovery of house dust fungi after the freezing step. A pooled stock of skin scales was obtained using beard shavings collected from electric razors of eight volunteers, and these scales were left untreated at room temperature before adding to the dust stock at the start of each experiment to provide a 1:1 (wt:wt) mixture. No yeast was added to this "natural" skin and dust diet.

To obtain eggs for developmental studies, 30 adult females were added to glass microculture vials as described above that contained either the liver and yeast diet or the pooled skin and dust diet. They were left at $25^{\circ} \mathrm{C}$ and $75 \% \mathrm{RH}$ until 50 eggs were laid. The females were then removed, and the eggs placed into the relevant hygrothermal conditions for the experi- ment. Observations were made daily for egg hatch and two to three times weekly for juvenile mortality and development.

Constant hygrothermal conditions of $25^{\circ} \mathrm{C}$ and 75 or $64 \% \mathrm{RH}$ were used in separate experiments to represent optimal laboratory rearing conditions and the lower relative humidity typical of a domestic environment, respectively. Relative humidity was controlled inside airtight plastic boxes by using saturated inorganic salt solution (Winston and Bates 1960), and it was verified periodically throughout experiments by using a relative humidity meter.

Principal components analysis (PCA; Legendre and Legendre, 1998) was implemented to assess correlations between response variables and to test for significant effects of predictor variables on the principal components, thereby avoiding inflation of type 1 errors. Provided the PCA showed a response variable was significantly affected by the treatment, it was assessed individually using analysis of variance (ANOVA) (Sokal and Rohlf, 1995). Data were log transformed to meet the assumptions of parametric tests: examination of residuals and fitted values showed that transformation was adequate to remove heteroscedasticity and non-normality of error variance. Significance was assumed at the $5 \%$ level $(P=$ $0.05)$, and a Gaussian error distribution was used.

\section{Results}

Adults. In the PCA, the first two principal components had eigenvalues greater than 1 and together captured $72 \%$ of the total variation in the response variables. Principal component $1(\mathrm{PCl})$ was positively correlated with reproductive period, female survival, fecundity, and male survival, whereas PC2 was positively correlated with prereproductive period and negatively correlated with reproductive rate. The pattern of high factor loadings on the same components suggests that the dependent variables are highly intercorrelated and that they are likely to show similar patterns among the ANOVAs.

In a multiple ANOVA using PCl as the dependent variable, the three-way interaction between strain, diet, and relative humidity was significant $(F=68.1$; $\mathrm{df}=7,71 ; P<0.001)$. All three manipulated variables were significant predictors of $\mathrm{PC} 2$, and the interaction between strain and RH was also significant $(F=19.8$; $\mathrm{df}=4,74 ; P<0.001)$. This suggests that the effects detected within each life history trait below were real and not artifacts of accepting random patterns as significant due to the number of separate tests done.

Fecundity. The three-way interaction between strain, diet, and relative humidity was significant $(F=$ 130.9 ; $\mathrm{df}=7,72 ; P<0.001)$. Fecundity was always higher at $75 \%$ compared with $64 \%$ RH for all strain and diet combinations (Tables 1 and 2). This difference was particularly striking on the liver and yeast diet at $64 \% \mathrm{RH}$, where fecundity was up to 25 times lower than at $75 \% \mathrm{RH}$ and up to 10 times lower than on the skin and dust diet at $64 \% \mathrm{RH}$. 
Table 1. Life history parameters of laboratory and wild populations of $D$. pteronyssinus (DP) reared on liver and yeast (lab) and skin and dust (dust) diets at $25^{\circ} \mathrm{C}$ and $75 \% \mathrm{RH}$

\begin{tabular}{|c|c|c|c|c|}
\hline & $\begin{array}{l}\text { Lab DP } \\
\text { lab diet }\end{array}$ & $\begin{array}{l}\text { Wild DP } \\
\text { lab diet }\end{array}$ & $\begin{array}{l}\text { Lab DP } \\
\text { dust diet }\end{array}$ & $\begin{array}{l}\text { Wild DP } \\
\text { dust diet }\end{array}$ \\
\hline \multicolumn{5}{|l|}{ Adults $(n=10)$} \\
\hline Total fecundity per female & $100 \pm 33.4$ & $66.9 \pm 15.9$ & $79.7 \pm 12.6$ & $60.9 \pm 15.9$ \\
\hline Prereproductive period (d) & $2.2 \pm 0.6$ & $2.9 \pm 1.1$ & $2.6 \pm 0.9$ & $8.3 \pm 3.4$ \\
\hline Reproductive period $(\mathrm{d})$ & $35.5 \pm 12.9$ & $43.1 \pm 22.9$ & $27.0 \pm 8.2$ & $35.9 \pm 18.9$ \\
\hline Rate of reproduction (eggs/female/d) & $3.0 \pm 1.1$ & $1.7 \pm 0.5$ & $3.2 \pm 1.0$ & $1.9 \pm 0.8$ \\
\hline Female survival $(\mathrm{d})$ & $45.2 \pm 13.8$ & $52.8 \pm 22.1$ & $39.9 \pm 17.5$ & $48.6 \pm 20.2$ \\
\hline Male survival $(\mathrm{d})$ & $34.8 \pm 14.4$ & $31.2 \pm 5.9$ & $33.9 \pm 21.1$ & $45.4 \pm 16.1$ \\
\hline \multicolumn{5}{|l|}{ Immatures $(n=50)$} \\
\hline$\%$ egg mortality & 0 & 0 & 0 & 0 \\
\hline Egg developmental time $(\mathrm{d})$ & $3.5 \pm 0.9$ & $4.5 \pm 0.8$ & $3.3 \pm 0.5$ & $5.4 \pm 2.8$ \\
\hline$\%$ juvenile mortality & 0 & 0 & 0 & 0 \\
\hline Juvenile developmental time $(\mathrm{d})$ & $9.8 \pm 1.2$ & $10.9 \pm 1.7$ & $13.1 \pm 0.7$ & $10.1 \pm 3.5$ \\
\hline Total egg-adult developmental time (d) & $13.3 \pm 1.4$ & $15.4 \pm 2.4$ & $16.4 \pm 0.7$ & $15.4 \pm 4.4$ \\
\hline
\end{tabular}

Results show mean $\pm \mathrm{SD}$.

At $75 \%$ RH (Table 1), there was no effect of diet on fecundity. The laboratory strain of mites, however, had significantly higher fecundity than the wild strain on both diets $(F=15.6$; $\mathrm{df}=1,38 ; P<0.001)$.

At $64 \%$ RH (Table 2), the interaction between strain and diet was significant $(F=86.0 ; \mathrm{df}=3,36 ; P<$ 0.001 ). Both mite strains had higher fecundity on the skin and dust diet than on the liver and yeast diet, but on the skin and dust diet the laboratory strain had the highest fecundity, whereas on the liver and yeast diet the wild strain had higher fecundity.

Prereproductive Period. The three-way interaction between strain, diet, and relative humidity was significant $(F=27.9 ; \mathrm{df}=7,72 ; P<0.001)$. Prereproductive period (defined here as the period between mating of female tritonymphs with males and production of first eggs) was shorter at $75 \% \mathrm{RH}$ than at $64 \% \mathrm{RH}$ for every combination of strain and diet (Tables 1 and 2). High relative humidity shortened prereproductive period to less than any group at low relative humidity, except for wild mites on the skin and dust diet.

At $75 \% \mathrm{RH}$ (Table 1), there was a significant interaction between strain and diet $(F=27.2 ; \mathrm{df}=3,36$; $P<0.001)$. On both diets, the laboratory mites had a shorter prereproductive period than the wild mites.
Wild mites had a significantly longer prereproductive period on the skin and dust diet compared with the liver and yeast diet $(F=32.0 ; \mathrm{df}=1,18 ; P<0.001)$, but no effect of diet was seen in laboratory mites.

At $64 \% \mathrm{RH}$ (Table 2), there were no significant differences between strains. In both strains, the skin and dust diet resulted in significantly longer prereproductive periods compared with the liver and yeast $\operatorname{diet}(F=18.9 ; \mathrm{df}=1,18 ; P<0.001)$.

Reproductive Period. The three-way interaction between strain, diet, and relative humidity was significant $(F=33.9 ; \mathrm{df}=7,72 ; P=0.008)$. On the liver and yeast diet, both strains of mites showed markedly shorter reproductive periods at $64 \% \mathrm{RH}$ compared with $75 \%(F=68.4 ; \mathrm{df}=2,36 ; P<0.001)$. There was no significant response to relative humidity in wild mites on the skin and dust diet, but in the laboratory strain fed on the skin and dust diet reproductive period was significantly longer at $64 \%$ than at $75 \% \mathrm{RH}$ $(F=5.9 ; \mathrm{df}=1,18 ; P=0.026) \quad($ Tables 1 and 2$)$.

There was no influence of diet on reproductive period at $75 \% \mathrm{RH}$. The only significant difference between strains was seen on the liver and yeast diet on which wild mites had a longer mean reproductive

Table 2. Life history parameters of laboratory and wild populations of $D$. pteronyssinus (DP) reared on liver and yeast (lab) and skin and dust (dust) diets at $25^{\circ} \mathrm{C}$ and $64 \% \mathrm{RH}$

\begin{tabular}{|c|c|c|c|c|}
\hline & $\begin{array}{l}\text { Lab DP } \\
\text { lab diet }\end{array}$ & $\begin{array}{l}\text { Wild DP } \\
\text { lab diet }\end{array}$ & $\begin{array}{l}\text { Lab DP } \\
\text { dust diet }\end{array}$ & $\begin{array}{l}\text { Wild DP } \\
\text { dust diet }\end{array}$ \\
\hline \multicolumn{5}{|l|}{ Adults $(n=10)$} \\
\hline Total fecundity per female & $3.8 \pm 0.9$ & $6.9 \pm 3.2$ & $48.0 \pm 10.3$ & $24.4 \pm 5.5$ \\
\hline Pre-reproductive period $(\mathrm{d})$ & $6.2 \pm 1.9$ & $6.0 \pm 1.6$ & $12.4 \pm 6.2$ & $10.1 \pm 3.7$ \\
\hline Reproductive period $(\mathrm{d})$ & $2.8 \pm 1.9$ & $6.3 \pm 3.5$ & $36.2 \pm 8.8$ & $29.4 \pm 10.3$ \\
\hline Rate of reproduction (eggs/female/d) & $2.3 \pm 1.7$ & $1.3 \pm 0.7$ & $1.4 \pm 0.6$ & $0.8 \pm 0.4$ \\
\hline Female survival $(\mathrm{d})$ & $10.2 \pm 2.4$ & $18.7 \pm 4.8$ & $56.6 \pm 13.1$ & $33.1 \pm 7.8$ \\
\hline Male survival (d) & $9.8 \pm 2.9$ & $11.6 \pm 4.0$ & $44.0 \pm 14.1$ & $37.9 \pm 12.8$ \\
\hline \multicolumn{5}{|l|}{ Immatures $(n=50)$} \\
\hline$\%$ egg mortality & 40 & 0 & 0 & 0 \\
\hline Egg development time (d) & $4.5 \pm 1.1$ & $8.0 \pm 0$ & $8.0 \pm 0$ & $2.3 \pm 1.0$ \\
\hline$\%$ juvenile mortality & 100 & 100 & 0 & 0 \\
\hline Juvenile development time (d) & & & $16.0 \pm 3.5$ & $23.9 \pm 9.8$ \\
\hline Total egg-adult development time (d) & & & $24.0 \pm 3.5$ & $26.0 \pm 9.9$ \\
\hline
\end{tabular}

Results show mean \pm SD. 
period than laboratory mites $(F=68.4 ; \mathrm{df}=2,36 ; P<$ 0.001) (Table 1).

At $64 \%$ RH (Table 2), the magnitude of the response to diet differed between the two strains of mites $(F=$ 35.8; $\mathrm{df}=3,36 ; P=0.003$ ). Although both strains of mites showed significantly shorter reproductive periods on the liver and yeast diet compared with the skin and dust diet, the response was greater in the laboratory strain. As found at $75 \% \mathrm{RH}$, wild mites had a longer mean reproductive period than laboratory mites on the liver and yeast diet. In contrast, on the skin and dust diet, laboratory mites had a significantly longer reproductive period than wild mites.

Reproductive Rate. Reproductive rate was higher at $75 \% \mathrm{RH}$ than at $64 \%$ in both mite strains and higher in the laboratory strain than in the wild strain at both relative humidity levels $(F=27.0 ; \mathrm{df}=2,77 ; P<0.001)$ (Tables 1 and 2). Diet did not affect reproductive rate.

Female Survival. Female survival (the period from mating of female tritonymphs with males and death) of both mite strains decreased at $64 \% \mathrm{RH}$ compared with $75 \% \mathrm{RH}$ on the liver and yeast diet $(F=57.8 ; \mathrm{df}=$ 3,$36 ; P<0.001$ ), and this decline in survival was more marked in the laboratory mite strain than in the wild strain. On the skin and dust diet, female survival increased with relative humidity in the wild strain, but it decreased with increasing relative humidity in the laboratory strain $(F=5.0 ; \mathrm{df}=3,37 ; P=0.005)$ (Tables 1 and 2).

Diet had no significant effect on female survival at $75 \% \mathrm{RH}$, and the only difference between strains at this relative humidity was found on the liver and yeast diet, on which survival of wild females was greater than laboratory-reared females $(F=57.8 ; \mathrm{df}=3,36$; $P<0.001$ ) (Table 1).

At $64 \%$ RH (Table 2), the two mite strains differed in the magnitude of their response to $\operatorname{diet}(F=71.9$; $\mathrm{df}=3,36 ; P<0.001$ ). Although female survival of both mite strains was lower on the liver and yeast diet compared with the skin and dust diet, the laboratory strain demonstrated a much greater increase in survival on the skin and dust diet than did the wild mites. Wild females on the liver and yeast diet had greater survival than laboratory mites on this diet, whereas the laboratory strain had much greater survival than the wild strain on the skin and dust diet.

Male Survival. The three-way interaction between mite strain, diet, and relative humidity was significant $(F=20.8 ; \mathrm{df}=7,71 ; P<0.001)$. Survival of males (survival time of males of unknown age during experiments) of both mite strains decreased at $64 \% \mathrm{RH}$ compared with $75 \%$ RH on the liver and yeast diet $(F=$ $125.3 ; \mathrm{df}=1,37 ; P<0.001)$. On the skin and dust diet, this effect was seen only in the wild mite strain $(F=$ $29.5 ; \mathrm{df}=3,35 ; P<0.001$ ), and it was less marked than that seen on the liver and yeast diet (Tables 1 and 2).

At $75 \%$ RH (Table 1), there was no effect of mite strain on male survival; however, wild males on the skin and dust diet had greater survival than those on the liver and yeast diet at this relative humidity $(F=$ $29.5 ; \mathrm{df}=3,35 ; P<0.001)$. At $64 \% \mathrm{RH}$ (Table 2$)$, again diet was the only significant predictor of male survival, with males of both strains on the skin and dust diet living longer than those on the liver and yeast $\operatorname{diet}(F=$ 125.3; $\mathrm{df}=1,37 ; P<0.001$ ).

Immature Development. Only PCl had an eigenvalue greater than 1 , and it explained $78.8 \%$ of the variation in egg, juvenile, and total development. PC1 was positively correlated with all of these factors, and it had high factor loadings of all, suggesting that the dependent variables are highly intercorrelated and likely to show similar patterns among the ANOVAs.

The significant interactions between strain and relative humidity and strain and diet in predicting PCl $(F=85.6 ; \mathrm{df}=5,255 ; P<0.001)$ indicate that the effects of strain on immature development are different at different levels of relative humidity and on different diets. These findings suggest that effects detected within each developmental trait were real and not artifacts of accepting random patterns as significant due to the number of separate tests done.

Egg Development. The three-way interaction between mite strain, diet, and relative humidity was significant $(F=91.1 ; \mathrm{df}=7,333 ; P<0.001)$. Compared with $75 \% \mathrm{RH}$, egg mortality at $64 \%$ was $40 \%$ higher in laboratory mites on the liver and yeast diet. On this diet, eggs of both strains of mites developed faster at $75 \% \mathrm{RH}$ compared with $64 \%(F=111.6$; $\mathrm{df}=7,333$; $P<0.001)$. However, on the skin and dust diet, although the laboratory strain showed more rapid egg development at $75 \%$ compared with $64 \%$, egg development in the wild strain was inhibited at $75 \% \mathrm{RH}(F=$ 100.6; $\mathrm{df}=7,333 ; P<0.001)($ Tables 1 and 2$)$.

At $75 \%$ RH (Table 1), there was no effect of diet on egg development. Eggs from the laboratory strain, however, developed more quickly than those laid by the wild strain on both diets $(F=52.7 ; \mathrm{df}=1,198 ; P<$ $0.001)$.

At $64 \%$ RH, both mite strain and diet influenced egg development (Table 2). Developmental times were quicker when the laboratory mite strain was on its accustomed diet (liver and yeast) compared with the skin and dust diet and also when the wild mites were on their accustomed diet (skin and dust) compared with the liver and yeast $\operatorname{diet}(F=194.3 ; \mathrm{df}=1,198 ; P<$ $0.001)$. Strain effects were seen on the liver and yeast diet where wild mite eggs had much slower development than eggs from the laboratory strain $(F=111.6$; $\mathrm{df}=7,333 ; P<0.001)$, whereas the reverse was true on the skin and dust diet.

Juvenile Development. No juveniles of either strain of mites completed development on the liver and yeast diet at $64 \% \mathrm{RH}$; thus, comparisons could be made between strain and relative humidity on the skin and dust diet only. At $75 \% \mathrm{RH}$, both strains had faster juvenile development than at $64 \%(F=70.4 ; \mathrm{df}=5$, 294; $P<0.001$ ) (Tables 1 and 2).

At $75 \% \mathrm{RH}$, juvenile development responded differently to diet between strains $(F=24.3 ; \mathrm{df}=3,196$; $P<0.001)$. Each strain had faster development on the diet to which they were accustomed compared with the alternative diet (Table 1). At 64\% RH on the skin and dust diet, the wild mites had slower juvenile de- 
velopment than the laboratory strain on this $\operatorname{diet}(F=$ 60.6; $\mathrm{df}=3,196 ; P<0.001$ ) (Table 2).

Total Development. Again, because no juveniles of either strain of mites completed development on the liver and yeast diet at $64 \% \mathrm{RH}$, comparisons were made between strain and relative humidity on the skin and dust diet only. On this diet, total development was faster at $75 \% \mathrm{RH}$ than at $64 \% \mathrm{RH}$ in both strains $(F=$ 72.3; $\mathrm{df}=4,295 ; P<0.001)$ (Tables 1 and 2).

At $75 \% \mathrm{RH}$ in the laboratory mite strain, the skin and dust diet markedly delayed total development compared with the liver and yeast diet, but no significant effect of diet was detected in the wild strain $(F=12.0$; $\mathrm{df}=1,198 ; P<0.001$ ) (Table 1$)$. At $64 \% \mathrm{RH}$ there were no significant differences in total development of the two mite strains on the skin/dust diet (Table 2).

\section{Discussion}

This study has provided life history parameters of laboratory-reared $D$. pteronyssinus on a laboratory diet at $25^{\circ} \mathrm{C}$ and $75 \% \mathrm{RH}$. The mites tested seemed to have higher reproductive parameters and faster development than those from previous reports (Spieksma 1967; Blythe 1976; Dobson 1979; Gamal-Eddin et al. 1983a, 1983b, 1983c; Ho and Nadchatram 1984; Colloff 1987a, 1987b; Andersen, 1988; Hart and Fain 1988; Arlian et al. 1990). Differences between these results are likely to be due to differences in strain of mites, diet, or both. We have demonstrated the importance of diet in this study, and we are also currently investigating the extent to which different strains of wild mites may vary in their life history parameters.

There have been few published studies on life history parameters of laboratory-reared D. pteronyssinus at $25^{\circ} \mathrm{C}$ and $64 \% \mathrm{RH}$. However, our laboratory mites seemed to perform less well at low relative humidity than those from previous reports (Gamal-Eddin et al. 1983a, 1983b, 1983c; Colloff 1987a, 1987b). This may be due to the diet of desiccated liver and yeast, which seemed to be unsuitable for mite reproduction and development at low relative humidity compared with the skin and dust diet (see below).

This study also has provided the first comprehensive data set of adult reproduction and immature survival and development of wild $D$. pteronyssinus in optimal and suboptimal rearing conditions. Previously, only egg survival and development have been reported by Colloff (1987a, 1987b), who suggested that eggs of wild mites survive better and develop more quickly than those of laboratory mites when reared in cool, dry conditions of temperature and relative humidity, whereas in warm, humid conditions the reverse is true. Our results also suggest that laboratory mites perform better (higher fecundity and rate of reproduction, shorter prereproductive period, and faster egg development) than wild mites in optimum rearing conditions $(75 \% \mathrm{RH})$, but in suboptimum conditions $(64 \%$ $\mathrm{RH}$ ), laboratory-reared mites perform less well (lower fecundity, shorter reproductive period, reduced female survival, and higher egg mortality) on the liver and yeast diet than wild mites. In contrast, on the skin and dust diet at low relative humidity, fecundity, rate of reproduction, reproductive period, and female survival of laboratory mites were higher than found in wild mites, but rearing the laboratory mites before the experiment on an optimized liver and yeast diet is likely to have had an influence on their subsequent egg production and survival on the skin and dust diet.

In some arthropods, specific traits can be selected after as little as five generations (Navarro et al. 1985, Yano and Takafuji 2002, Young et al. 2003). Therefore, during the period between collection and the start of experiments, it is possible that our wild mite cultures may have, in part, adapted to laboratory culture conditions and thus represent an intermediate stage between wild and fully adapted long-term laboratory cultures. However, this seems unlikely, because our wild cultures were reared in conditions relating very closely to those found in the home (diurnally fluctuating hygrothermal conditions) and on a natural diet consisting of only skin scales and house dust.

The poor performance of adults and immatures on the liver and yeast diet compared with the skin and dust diet at low relative humidity was particularly striking. Most existing data on reproduction and development of house dust mites have been obtained from mites reared on laboratory diets that are highly nutritious and that provide good population development in optimum hygrothermal conditions, but such diets may not provide an ideal substrate for mite survival at low relative humidity. This could explain the sparse data on survival, reproduction, and development of house dust mites reared at relative humidities below 75\%. However, de Saint Georges-Gridelet (1984) previously reported high population growth of D. pteronyssinus at $64 \% \mathrm{RH}$ on diets high in lipids. In this study, lipids present in skin scales in the house dust substrate could explain the survival of mites in their natural habitat where hygrometric conditions are often below the critical equilibrium activity (Arlian 1975, Arlian and Veselica 1981) of the mites. Our results seem to agree with this hypothesis, and they suggest that data on mite performance on laboratory yeast-based diets, particularly in suboptimal conditions, are unlikely to represent performance on a skinbased diet in their natural dust habitat.

Population models to predict dust mite populations in homes are currently under development by using previously published data primarily from laboratory populations of mites reared on laboratory diets (Pretlove et al. 2001, 2005; Crowther et al. 2006; Biddulph et al. 2007). The current study has highlighted the requirement for a more comprehensive data set from wild mite populations reared on a natural diet for use in these models. Work is underway by the current authors to provide these data.

Another critical factor likely to have an influence on the life history parameters of house dust mites is fluctuating temperature and relative humidity. De Boer et al. (1998) have shown that D. pteronyssinus can survive and produce eggs when held at low relative humidity and given as little as $3 \mathrm{~h}$ of moist air per day. Arlian et al. (1999) suggested that the development of 
Dermatophagoides farinae (Hughes) was slower in fluctuating relative humidity compared with constant high relative humidity. More recently, Pike et al. (2005) found that the population dynamics for $D$. pteronyssinus were similar in both fluctuating and constant conditions of temperature and relative humidity. However, Colloff (1987a) proposed that laboratory mite populations were less able to withstand diurnal fluctuations in microclimate than wild populations of mites. This is also the subject of a subsequent article by us using wild mites reared on a natural diet.

\section{Acknowledgments}

We thank Paul Johnson and Lucy Tallents (Department of Zoology, University of Oxford, Oxford, United Kingdom) for statistical advice. This study was funded by UK Engineering and Physical Sciences Research Council grants GR/ S70661/01 and GR/S70678/01.

\section{References Cited}

Andersen, A. 1988. Population growth and developmental stages of the house dust mite, Dermatophagoides pteronyssinus (Acari: Pyroglyphidae). J. Med. Entomol. 25: 370373.

Arlian, L. G. 1975. Dehydration and survival of the European house dust mite, Dermatophagoides pteronyssinus. J. Med. Entomol. 12: 437-442.

Arlian, L. G., and M. M. Veselica. 1981. Re-evaluation of the humidity requirements of the house dust mite Dermatophagoides farinae (Acari: Pyroglyphidae). J. Med. Entomol. 18: 351-352.

Arlian, L. G., J. S. Neal, and D. Vyszenski-Moher. 1999. Fluctuating hydrating and dehydrating relative humidities effects on the life cycle of Dermatophagoides farinae (Acari: Pyroglyphidae). J. Med. Entomol. 36: 457-461.

Arlian, L. G., C. M. Rapp, and S. G. Ahmed. 1990. Development of Dermatophagoides pteronyssinus (Acari: Pyroglyphidae). J. Med. Entomol. 27: 1035-1040.

Biddulph, P., D. Crowther, B. Leung, T. Wilkinson, B. Hart, T. Oreszczyn, S. Pretlove, I. Ridley, and M. Ucci. 2007. Predicting the population dynamics of the house dust mite Dermatophagoides pteronyssinus (Acari: Pyroglyphidae) in response to a constant hygrothermal environment using a model of the mite life cycle. Exp. Appl. Acarol. 41: 61-86.

Blythe, M. E. 1976. Some aspects of the ecological study of the house dust mite. Br. J. Dis. Chest 70: 3-31.

Colloff, M. J. 1987a. Differences in development time, mortality and water loss between eggs from laboratory and wild populations of Dermatophagoides pteronyssinus. Exp Appl. Acarol. 3: 191-200.

Colloff, M. J. 1987b. Effects of temperature and relative humidity on development times and mortality of eggs from laboratory and wild populations of the European house dust mite Dermatophagoides pteronyssinus (Acari: Pyroglyphidae). Exp. Appl. Acarol. 3: 279-289.

Crowther, D., T. Wilkinson, P. Biddulph, T. Oreszczyn, S. Pretlove, and I. Ridley. 2006. A simple model for predicting the effect of hygrothermal conditions on populations of house dust mite Dermatophagoides pteronyssinus (Acari: Pyroglyphidae). Exp. Appl. Acarol. 79: 127-148.

Cunningham, M. J. 1999. Modelling of some dwelling internal microclimates. Bldg. Environ. 34: 523-536.

De Boer, R., K. Kuller, and O. Kahl. 1998. Water balance of Dermatophagoides pteronyssinus (Acari: Pyroglyphidae) maintained by brief daily spells of elevated air humidity. J. Med. Entomol. 35: 905-910.

de Saint Georges-Gridelet, D. 1984. Effects of dietary lipids on the population growth of Dermatophagoides pteronyssinus. Acarology 6: 351-357.

Dobson, R. M. 1979. Some effects of microclimate on the survival and development of Dermatophagoides pteronyssinus (Trouessart). Acarologia 2: 482-486.

Gamal-Eddin, F. M., F. M. Abou-Sinna, S. E. Tayel, A. M. Aboul-Atta, A. M. Seif, and S. M. Gaafar. 1983a. Duration of the developmental stages of house dust mites Dermatophagoides farinae and D. pteronyssinus under controlled temperatures and relative humidities to pave the way in front of the workers in the field of house dust mite asthmatic bronchitis. 1. Pre-imaginal period. J. Egypt. Soc. Parasitol. 13: 319-334.

Gamal-Eddin, F. M., K. K. Shehata, F. Abou-Sinna, S. Tayel, A. M. Aboul-Atta, B. A. El-Ahmedaawy, M. A. Fayed, A. H. Hafez, and M. Imam. 1983b. Duration of the development stages of house-dust mites Dermatophagoides farinae and D. pteronyssinus under controlled conditions to pave the way in front of the workers in the field of house-dust mite asthmatic bronchitis. 2. Oviposition period, fecundity and oval duration. J. Egypt. Soc. Parasitol. 13: $557-581$.

Gamal-Eddin, F. M., K. K. Shehata, F. M. Abou-Sinna, S. E. Tayel, A. M. Aboul-Atta, B. A. El-Ahmedaawy, M. A. Fayed, A. H. Hafez, and M. Imam. 1983c. Duration of the developmental stages of house dust mites Dermatophagoides farinae and D. pteronyssinus under controlled conditions to pave the way in front of the workers in the field of house dust mite asthmatic bronchitis 3. Larval duration. J. Egypt. Soc. Parasitol. 13: 583-595.

Hart, B. J., and A. Fain. 1988. Morphological and biological studies of medically important house-dust mites. Acarologia 29: 285-295.

Ho, T.M., and M. Nadchatram. 1984. Life cycle and survival of Dermatophagoides pteronyssinus (Trouessart) (Acarina: Astigmata: Pyroglyphidae) in a tropical laboratory. Trop. Biomed. 1: 159-162.

Legendre, P., and L. Legendre. 1998. Numerical ecology, 2nd ed. Elsevier, Amsterdam, The Netherlands.

Navarro, S., R. Dias, and E. Donahaye. 1985. Induced tolerance of Sitophilus oryzae adults to carbon dioxide. J. Stored-Prod. Res. 21: 207-213.

Pike, A. J., M. J. Cunningham, and P. J. Lester. 2005. Development of Dermatophagoides pteronyssinus (Acari: Pyroglyphidae) at constant and simultaneously fluctuating temperature and humidity conditions. J. Med. Entomol. 42: 266-269.

Pretlove, S., I. Ridley, J. Horwood, B. Leung, P. Cox, D. Thomson, N. Baker, D. Crowther, and T. Oreszczyn. 2001. A combined transient hygrothermal and population model of house dust mites in beds. In Proceedings of IAQ 2001: Moisture, Microbes and Health Effects: Indoor Air Quality and Moisture in Buildings, American Society of Heating, Refrigeration and Airconditioning Engineers (ASHRAE), 4-7 November 2001, San Francisco, CA. ASHRAE, Atlanta, GA.

Pretlove, S., R. Critchley, R. Howard, and T. Oreszczyn. 2002. The Nottingham energy, health and housing study: reducing relative humidity, dust mites and asthma. Bldg. Serv. Eng. Res. Technol. 23: 43-55.

Pretlove, S., T. Oreszczyn, I. Ridley, T. Wilkinson, and D. Crowther. 2005. A steady state model for predicting hygrothermal conditions in beds in relation to house dust mite requirements. Bldg. Serv. Eng. Res. Technol. 26: 301-314. 
Sokal, R. R., and F. J. Rohlf. 1995. Biometry. W.H. Freeman New York.

Spieksma, F.Th.M. 1967. The house dust mite Dermatophagoides pteronyssinus (Trouessart, 1897). Ph.D. dissertation, University of Leiden, Leiden, The Netherlands.

Winston, P. W., and D. H. Bates. 1960. Saturated solutions for the control of humidity in biological research. Ecology 41: 232-237.

Yano, S., and A. Takafuji. 2002. Variation in the life history pattern on Tetranychus urticae (Acari: Tetranychi- dae) after selection for dispersal. Exp. Appl. Acarol. 27: $1-10$.

Young, H. P., D. W. Bailey, and R.M.R.M. Roe. 2003. Spinosad selection of a laboratory strain of the tobacco budworm, Heliothis virescens (Lepidoptera: Noctuidae), and characterization of resistance. Crop Prot. 22: 265-273.

Received 22 September 2006; accepted 19 March 2007. 